(Aus dem pharmakologischen Institut der Universität Heidelberg.)

\title{
Versuche \\ am überlebenden Dünndarm von Säugetieren.
}

\author{
VI. Mitteilung. \\ Von
}

R. Magnus.

(Mit 4 Textigguren.)

Zu meinem grossen Bedauern bin ich genötigt, mich mit den Ausführungen eines Forschers auseinanderzusetzen, welcher nicht mehr unter den Lebenden weilt. In dem kürzlich erschienenen Supplementband seines Archives veröffentlicht Engelmann eine nacbgelassene Arbeit von Paul Schulz, ,Über die angebliche refraktäre Periode der Darmmuskulatur der Warmblïter" ${ }^{\text {1), in }}$ welcher über eine Nachprüfung meiner Versuche an plexushaltigen Längsmuskelstreifen des Katzendünndarms berichtet wird. Schulz kommt zu dem Ergebnis, dass eine refraktäre Periode an diesen Objekten sich nicht nachweisen lässt.

Ich habe es nicht für überflüssig erachtet, auch meinerseits diese Versuche wieder aufzunehmen. Das Ergebnis ist, dass ich meine frühere Feststellung vollständig aufrechterhalten muss. An tadellosen, gut erregbaren Präparaten lässt sich immer eine refraktäre Periode nachweisen. Die möglichen Ursachen für diese Differenz unserer tatsächlichen Befunde hoffe ich im folgenden aufklären zu können.

$\mathrm{Zu}$ den Versuchen dienten wieder Katzen, welche in tiefer Äthernarkose durch Nackenschlag getötet wurden. Nach schneller Eröffnung der Bauchböhle wurde eine Darmschlinge hervorgezogen und mit einer spitzen und tadellos blanken Präpariernadel die Längsmuskulatur an der dem Mesenterialansatz gegenüberliegenden Seite

1) Arch. f. Physiol. 1905 Suppl. S. 23. 
abgehoben. Durch einfachen Zug liess sich dann ein 'genügend langes Stück der Längsmuskulatur gewinnen; in einzelnen Fällen liessen sich zusammenhängende Streifen vom Duodenum bis fast zur Bauhin'sehen Klappe abziehen. Die Präparate werden in eine Schale mit körperwarmer Ringer'scher Flüssigkeit gebracht, durch welche Sauerstoff perlt. Nach einiger Zeit beginnen Spontanbewegungen; man sucht sich nun zum eigentlichen Versuch solche Partien aus, welche besonders lebhafte Bewegungen zeigen. Ein geeigneter Streifen von 3-6 cm Länge wird in der schon (Pflüger's Arch. Bd. 102 S. 127 Fig. 1) angegebenen Weise mit dem Schreibhebel verbunden. Die Hebelvergrösserung war in allen Versuchen dreifach, die auf das Präparat wirkende Last betrug $0,8-1,5 \mathrm{~g}$. Derartige Muskelstreifen zeigen nun nach kurzer Zeit eine ausserordentlich starke Erschlaffung. (Präparate mit stärkerem Tonus sind unbrauchbar.) Die nach einiger Zeit einsetzenden Spontanbewegungen sind meist sehr ausgiebig. Zur Reizung diente der elektrische Strom von Léclanché-Elementen oder Akkumulatoren. Die Elektroden habe ich nicht mehr, wie in den früheren Versuchen, an dem Muskelstreifen selber angebracht, da hierdurch, wie Schulz richtig bemerkt hat, eine Schädigung gesetzt wird. Es tauchten vielmehr die beiden Elektroden rechts und links von dem Muskelstreifen in die Flüssigkeit, so dass der Muskel ungefähr sich in der Mitte 2 wischen beiden befand. Der Elektrodenabstand betrug durehsehnittlich $3-4 \mathrm{~cm}$. Bei dieser Anordnung sind natürlich stärkere Ströme erforderlich, als wenn eine Elektrode am Muskel liegt, weil die Flüssigkeit eine sehr ausgiebige Nebenschliessung bildet und nur ein kleiner Teil des Stromes auf den Muskel wirksam wird. Als Elektroden dienten entweder zwei Platindrähte oder unpolarisierbare Elektroden aus Zink, Zinksulfat und Kochsalzton. Bei den Platinelektroden war bei meiner Anordnung der Strom yon 4-6 Léclanché-Elementen notwendig, um sichere Reaktionen zu erzielen. Bei Anwendung der unpolarisierbaren Elektroden mussten noch bis zu sechs Akkumulatoren hinzugenommen werden ${ }^{1}$ ).

Für die erhaltenen Resultate werden im nachfolgenden noch-

1) Schulz scheint angenommen za haben, dass ich bei meinen früheren Versuchen mich besonders starker elektrischer Reize bedient habe. Dies ist ein: Irrtam; die benufaten Ströme waren stets die schwächsten, welche noch sichere Reaktionen erzeugten. 
mals einige charakteristische Kurvenbeispiele gegeben werden. Nach Schluss jedes Versuches wurde jedes Präparat histologisch auf das Vorhandensein und die Vollständigkeit des Auerbach'schen Plexus untersucht. Hierzu bedient man sich am besten der Methylenblaufärbung in einer mir vom Professor Langl e y freundlichst mitgeteilten Form. Die Präparate kommen für $1 / 2$ Stunde in eine Lösung von $0,06 \%$ Methylenblau in $0,6 \%$ Kochsalzlösung, dann werden sie in einigen Tropfen dieser Lösung auf dem Objektträger ausgebreitet (Plexusseite nach oben), bleiben ohne Deckglas einige Minuten an
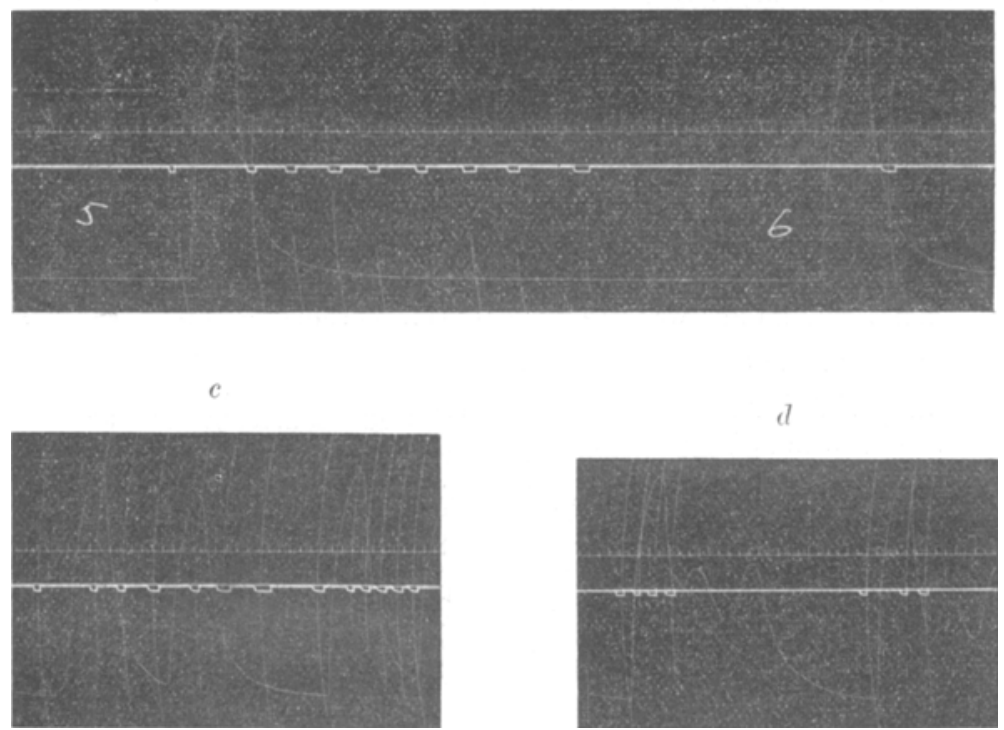

Fig. 1. Abgezogener Längsmuskelstreif. Auerbach'scher Plexus völlig intakt. Platinelektroden. a 5 Flemente. $b, c 6$ Elemente. $d$ Do. Elektroden genähert. (Markierung der Reizmomente bei stillstehender Trommel.) (Auf $1 / 2$ verkleinert.)

der Luft liegen und werden sodann in diesem Zustand bei schwacher und mittlerer Vergrösserung untersucht. Für die erhaltenen Resultate führe ich folgende Kurvenbeispiele an: Fig 1 zeigt die Bewegungen eines sebr spärliche Spontankontraktionen ausführenden Präparates. Der Strom von fünf Elementen ruft eine lebhafte Kontraktion ( $a$ ) hervor, ist aber selbst in der zweiten Hälfte der Dekreszente und pach völliger Erschlaffung noch eine Zeitlang ausserstande, eine neue Kontraktion zu bewirken. Darauf wurde der Strom auf sechs Elemente verstärkt. Bei der nächsten Spontankontraktion (b) ist 
die Reizung gegen Ende der Dekreszente noch wirkungslos. Die nächste Kontraktion (c), welche durch elektrische Reizung bedingt ist, zeigt, dass das Präparat vom Beginn bis gegen das Ende der Dekreszente
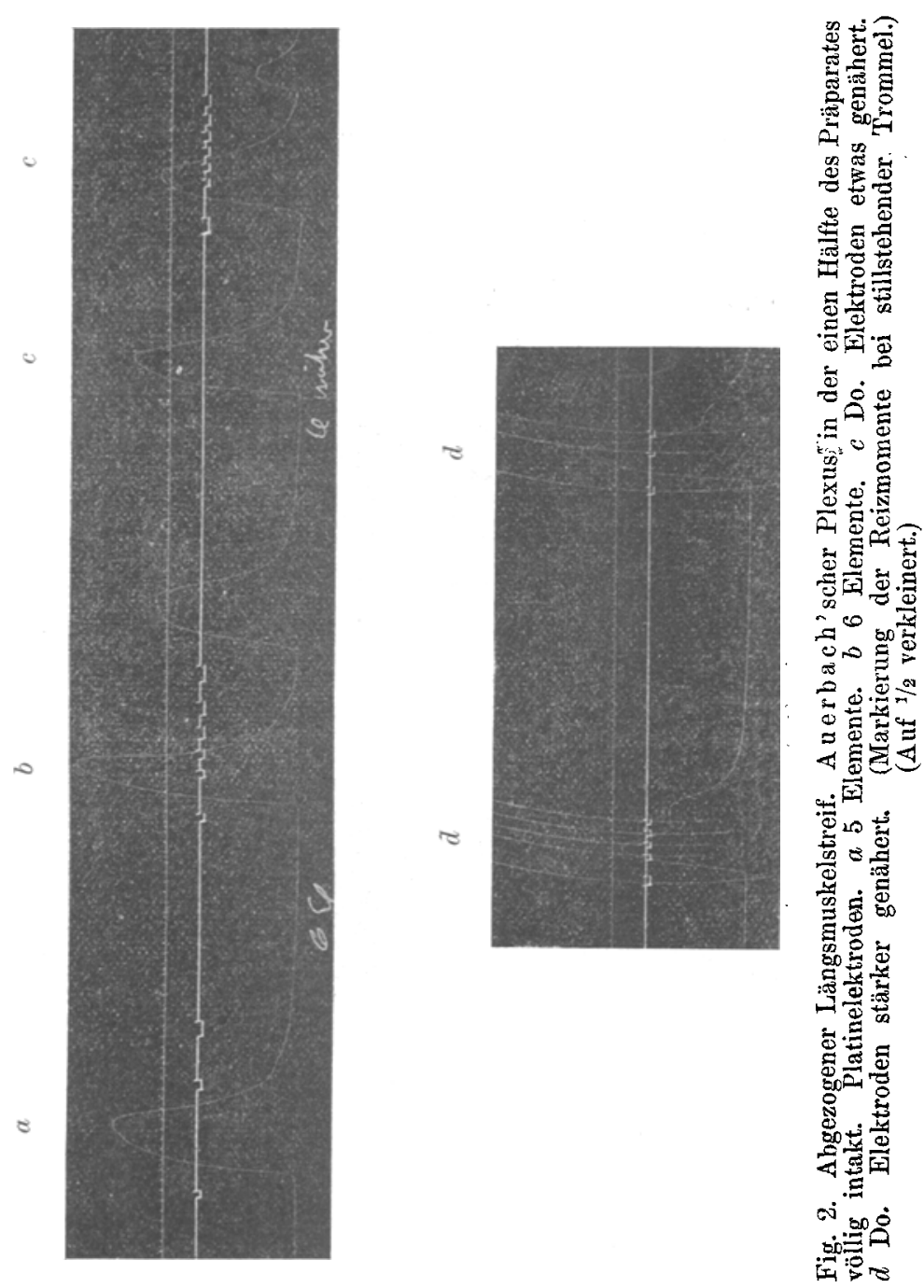

sich refraktär verhält und erst nach fast völliger Erschlaffung wieder anspricht. Dasselbe zeigen die folgenden Kontraktionen. Darauf wurden die Elektroden in der Flüssigkeit einander genähert und dadurch der Strom verstärkt. Man sieht jetzt bei den darauffolgenden beiden Kontraktionen (d), dass der Reiz während der Kreszente, 
auf dem Gipfel und im Beginn der Dekreszente wirkungslos bleibt, dagegen im weiteren Verlauf der Dekreszente (im zweiten Drittel) wirksam wird. Der Versuch zeigt, dass das Präparat eine deutliche refraktäre Periode besitzt, und dass die Erregbarkeit für stärkere Ströme eher wiederkehrt als für schwächere. Es herrschen also ähnliche Verhältnisse wie beim Herzen, welches ebenfalls seine volle Erregbarkeit im Verlauf der Dekreszente und nach der Ersehlaffung allmäblich wieder erhält (Engelmann). Die histologische Untersuchung ergab in diesem Falle, dass der A uerbach'sche Plexus vôllig intakt war.

Einen ganz entsprechenden Versuch gibt Fig. 2 wieder. Auch hier lässt sich die refraktäre Periode bei fünf Elementen $(a)$ bis nach

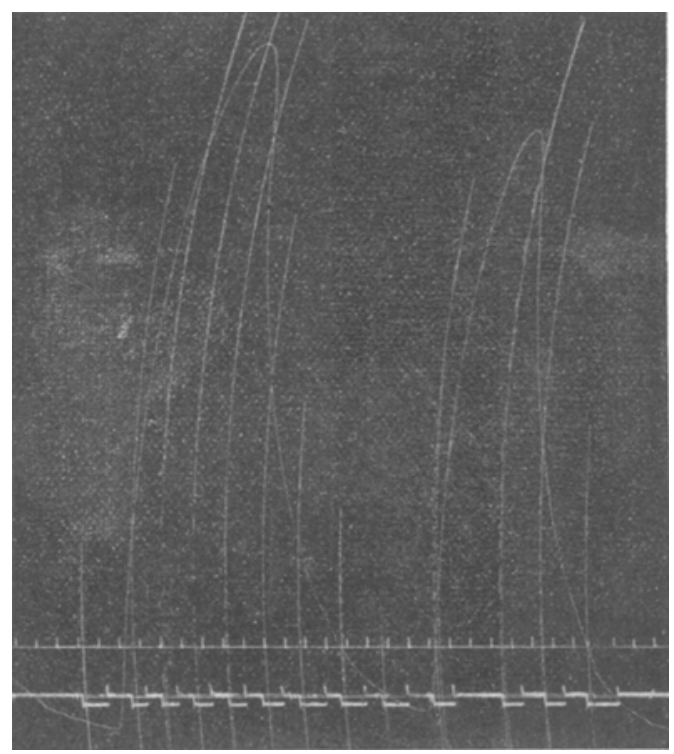

Fig. 3. Abgezogener Längsmuskelstreif. A u erbach'scher Plexus völlig intakt. Unpolarisierbare Elektroden. 6 Leclanché-Elemente 'und 6 Akkumulatoren. (Markierung der Reizmomente bei stillstehender Trommel.)

vollendeter Erschlaffung, bei stärkerem Strome bis zum Ende der Dekreszente $(c)$ und nach weiterer Verstärkung des Stromes $(d)$ jedenfalls bis zum Beginne der Dekreszente nachweisen. In diesem Falle war der A uerbach'sche Plexus in der einen Hälfte des Präparates ganz vollständig; und zwar auf der Seite, welche zwisehen den Elektroden gelegen war. In der anderen Hälfte zeigte der Plexus Lücken. Fig. 3 
zeigt einen Versuch mit unpolarisierbaren Elektroden. Hier sieht man die refraktäre Periode bis zum Ende dèr Dekreszente dauern. In anderen Fällen, in denen die histologische Untersuchung ergab, dass der Auerbach'sche Plexus intakt oder wenigstens in ziemlicher Vollständigkeit erhalten war, ergab sich, dass die refraktäre Periode bei der gewählten Reizstärke bis zur Mitte der Dekreszente oder bis zur Grenze des ersten und zweiten Drittels reichte, wie ich das schon in der vierten Mitteilung ${ }^{1}$ ) beschrieben und abgebildet habe.

Ich habe mich nicht damit begnügt, mich von neuem davon zu überzeugen, dass an guten Präparaten eine refraktäre Periode nachweisbar ist, sondern habe mir auch die Frage vorgelegt, weshalb $\mathrm{Schulz}$ zu abweichenden Ergebnissen gelangen konnte. Um letzteres zu verstehen, müssen wir zunächst einige Feblerquellen besprechen, welche bei derartigen Versuchen in Betracht kommen.

Zunächst bedarf es zur Herstellung der Präparate einiger Übung. Als ich die in dieser Mitteilung besebriebenen Versuche wieder aufnahm, hatte ich auch anfangs einige Misserfolge, die bei zunehmender Übung wegblieben. Die Präparate zeigten bei der histologischen Untersuchung einen sebr unvollständigen Auerbach'schen Plexus oder gar nur noch Trümmer desselben. Solche Objekte zeigten dann, dass die refraktäre Periode bei der gewählten Rejzstärke nur bis zum Gipfel dauerte, oder dass man sogar während der Dauer der Kreszente schon Extrazuckungen erbielt, wie es auch in den von Schulz abgebildeten Fällen zu sehen ist. Vor allem ist bei der Präparation auf eine tadellose Beschaffenheit der verwendeten Präpariernadel zu achten; sowie diese Unebenheiten oder rostige Stellen zeigt, wird der Auerbach'sche Plexus fast immer geschädigt. Besondere Aufmerksamkeit muss darauf verwendet werden, wie das Präparat aufgehängt ist. Hat man zu breite Streifen von Längsmuskulatur, so kann es vorkommen, dass sich nur die eine Hälfte kontrahiert, während die andere schlaff bleibt, wie dies Fig. 4 andeutet. In diesem Falle kann man bei elektrischer Reizung natürlich

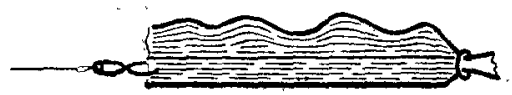

Fig, 4. in jedem Momente der Kreszente und Dekreszente eine Extrazuckung hervorrufen, welche, wie der Augensehein lehrt, dureh Kontraktion der schlaff gebliebenen Muskelhälfte bedingt ist. Man tut deshalb

1) Pflüger's Arch. Bd. 103 S. 525. 1904. (Fig. 4 und 5.) 
gut, nicht zu breite Präparate zu wählen und bei jedem Versuche auf den angegebenen Umstand besonders zu achten.

Ich glaube aus folgenden Gründen, dass Schulz in seinen Versuchen es mit schlecht erregbaren geschädigten Präparaten zu tun hatte. Erstens bildet er auf Fig. 8 seiner Abhandlung einen Fall $a b$, in dem er auf rasch wiederholte elektrische Reizung einen glatten Tetanus bekam. Ich habe ein derartiges Verhalten nur bei plexusfreien oder durch die Präparation schwer geschädigten plexushaltigen Muskelstreifen beobachtet und auf Fig. 9 der vierten Mitteilung einen Fall abgebildet, in dem das Präparat, das anfangs ebenfalls tetanisierbar war, sich im Laufe des Versuches so weit von der Schädigung durch die Präparation erholte, dass es auf denselben Reiz rhythmische Bewegungen zeigt ${ }^{1}$ ). Der zweite Grund, weshalb ich annehme, dass $\mathrm{Sehulz}$ es mit geschädigten Präparaten zu tun gehabt hat, ist, dass er mir vorwirft, ich habe den Tonus meiner Präparate nicht genügend berücksichtigt. Nun ist gerade eines der wesentlichsten Charakteristica der benutzten abgezogenen Längsmuskelstreifen, wenn sie gut hergestellt sind und einen ungeschädigten Auerbach'schen Plexus besitzen, die ausserordentliche Dehnbarkeit und der fast völlige Mangel jeder tonischen Verkürzung. Kurze Zeit, nachdem ein solches Muskelstück mit dem Hebel verbunden ist, beginnt es sich unter dem Einfluss seiner geringen Belastung $(0,8-1,5 \mathrm{~g})$ alsbald $\mathrm{zu}$ strecken, und es ist erstaunlich, bis zu welchem Grade diese Erschlaffung meistens geht. Ich habe stets gefunden, dass, wenn das Präparat tonisch verkürzt bleibt, es sich um ein schwer geschädigtes Objekt handelte.

Im übrigen hat $\mathrm{Schulz}$ selbst einen Fall abgebildet, in welcbem die refraktäre Periode wenigstens bis zum Gipfel der Kreszente dauerte (Fig. 9 S. 30). Er selber meinte freilich, das sei nur scheinbar, weil die Trommelgeschwindigkeit des Kymographions in diesem seinem Versuche zu gering gewesen sei, so dass man einen Knick in der Kurve während der Kreszente nicht zu sehen brauche, während ein solcher tatsächlich vorhanden sei. Schulz hat übersehen, dass es ein sehr einfaches Mittel gibt, um diese Hypothese auf ihre Richtigkeit zu prüfen. Man braucht nämlich die Kurven

1) Auch in den anderen angeführten Versuchen hat Schulz auf Dauerreiz oder frequente rhythmische Reizung immer nur mehr oder weniger unvollständige Tetani, nie gute rhythmische Bewegungen erhalten. 
nur durch eine Zylinderlinse mit senkreeht gehaltener Achse zu betrachten und auf diese Weise optisch in der Richtung der Abszisse auseinander zu ziehen, um genau denselben Effekt zu erreichen, als wenn die Trommelgeschwindigkeit die gewünschte grössere gewesen wäre. Betrachtet man die genannte Schulz'sche Kurve durch einen Zylinder von etwa zwölf Dioptrien ${ }^{\mathbf{l}}$ ), so sieht man ohne weiteres, dass von dem postulierten Knick nicht die Rede ist, und dass die Kurven in der Kreszente völlig glatt verlaufen. Ebenso kann man sich mit diesem einfachen optischen Hilfsmittel obne weiteres überzeugen, dass auf den Kurven, welche ich in Fig. 4 und 5 Seite 531 und 533 meiner vierten Mitteilung abgebildet habe, ebenso wie auf den Kurven, welche der gegenwärtigen Mitteilung beigegeben sind, von einer derartigen Täuschung durch zu geringe Trommelgeschwindigkeit nichts zu sehen ist.

Dass Präparate, welche einen schwer geschädigten nur sehr unvollständigen Auerbach'schen Plexus besitzen, noch imstande sind, Spontanbewegungen zu zeigen, darf uns nicht wundernehmen. Hierzu genügen oft schon geringe Reste, und es ist das der Grund, weshalb es einigermassen schwierig ist, wirklich zentrenfreie Präparate der Ringmuskulatur herzustellen. Ich habe diese Schwierigkeiten in der fünften Mitteilung ausdrücklich betont und gezeigt, dass man besonders bei der Anwendung erregender Gifte sehr oft von derartigen Präparaten noch rhythmische Bewegungen erhält (vgl. Pflüger's Arch. Bd. 108 S. 9 Fig. 1). Bei gesehädigten abgezogenen Längsmuskelstreifen, welche nur noch Reste des Auerbach'schen Plexus enthalten, genügen diese Reste zur Erzeugung von Spontanbewegungen. Es sind aber ausserdem in solchen Präparaten so viel Muskelpartien vorhanden, denen der A u erbach'sche Plexus fehlt, dass es nicht wundernehmen kann, wenn hier die refraktäre Periode vermisst wird.

Ich hatte in der früheren Mitteilung angegeben, dass es bei besonders gut erregbaren Präparaten gelingt, die refraktäre Periode auch mit Hülfe der mechanischen Reizung nachzuweisen. Schulz meint, es dürfte schwer sein, solehe Versuche beweisend zu gestalten; wenn man aber wirklich es mit gut erregbaren Objekten mit ungeschädigtem Auerbach'schen Plexus zu tun hat, wird man ge-

1) Man erhält bei jedem Optiker planzylindrische Gläser von 6 Dioptrien. Zwei mit den ebenen Flächen zusammengelegt, geben den gewünschten Frfolg. 
legentlich auf Präparate stossen, welche schon auf die einfache Berührung mit einer Nadel prompte Kontraktion zeigen, und an diesen das Phänomen der refraktären Periode nachweisen können.

Ich glaube im vorstehenden gezeigt zu haben, dass die refraktäre Periode der Darmmuskulatur, solange sie mit ihren Zentren verbunden ist, sich an gut erregbaren Präparaten mit nicht zu stark geschädigtem Auerbach'schen Plexus tatsächlich nachweisen lässt. 